MPP-2010-50

\title{
NEUTRINO DECAY INTO FERMIONIC QUASIPARTICLES IN LEPTOGENESIS
}

\author{
CLEMENS P. KIEßIG* ${ }^{\star}$ MICHAEL PLÜMACHER $\odot$ \\ Max-Planck-Institut für Physik (Werner-Heisenberg-Institut), Föhringer Ring 6 \\ D-80805 München, Germany \\ *E-mail: ckiessig@mpp.mpg.de, \\ ${ }^{\circ}$-mail: pluemi@mpp.mpg.de \\ MARKUS H. THOMA ${ }^{\dagger}$ \\ Max-Planck-Institut für extraterrestrische Physik, Giessenbachstraße, \\ D-85748 Garching, Germany \\ $\dagger^{\dagger}$-mail: mthoma@mpe.mpg.de
}

\begin{abstract}
We calculate the decay rate of the lightest heavy Majorana neutrino in a thermal bath using finite temperature cutting rules and effective Green's functions according to the hard thermal loop resummation technique. Compared to the usual approach where thermal masses are inserted into the kinematics of final states, we find that deviations arise through two different leptonic dispersion relations. The decay rate differs from the usual approach by more than one order of magnitude in the temperature range which is interesting for the weak washout regime. This work summarizes the results of Ref. 1 to which we refer the interested reader.

Keywords: Leptogenesis; Thermal field theory; Finite temperature field theory; Hard thermal loop; Plasmino.
\end{abstract}

\section{Introduction}

Leptogenesis ${ }^{2}$ is an extremely successful theory in explaining the baryon asymmetry of the universe by adding three heavy right-handed neutrinos $N_{i}$ to the standard model,

$$
\delta \mathcal{L}=i \bar{N}_{i} \partial_{\mu} \gamma^{\mu} N_{i}-\lambda_{\nu, i \alpha} \bar{N}_{i} \phi^{\dagger} \ell_{\alpha}-\frac{1}{2} M_{i} \bar{N}_{i} N_{i}^{c}+\text { h.c. },
$$

with masses $M_{i}$ at the scale of grand unified theories (GUTs) and Yukawa couplings $\lambda_{\nu, i \alpha}$ similar to the other fermions. This also solves the problem of the light neutrino masses via the see-saw mechanism without fine-tuning ${ }^{[3}$

The heavy neutrinos decay into lepton and Higgs boson after inflation, the decay is out of equilibrium since there are no gauge couplings to the standard model. If the CP asymmetry in the Yukawa couplings is large enough, a lepton asymmetry is created by the decays which is then partially converted into a baryon asymmetry by sphaleron processes. As temperatures are high, interaction rates and the $\mathrm{CP}$ 
asymmetry need to be calculated using thermal field theory ${ }^{4}$ rather than vacuum quantum field theory.

\section{Hard Thermal Loops and Thermal Masses}

When using bare thermal propagators in TFT,, 5 one can encounter IR singularities and gauge dependent results. In order to cure this problem, the hard thermal loop (HTL) resummation technique has been invented $6 / 7$ If $g$ is the coupling to the thermal bath, then for soft momenta $K \lesssim g T$, resummed propagators are used. For a scalar field e.g. this reads

$$
i \Delta^{*}=i \Delta+i \Delta(-i \Pi) i \Delta+\cdots=\frac{i}{\Delta^{-1}-\Pi}=\frac{i}{Q^{2}-m_{0}^{2}-\Pi} .
$$

The self energy $\Pi \sim g T$ then acts as a thermal mass $m_{\mathrm{th}}^{2}=\Pi$ and gives a correction $m_{\mathrm{tot}}^{2}:=m_{0}^{2}+m_{\mathrm{th}}^{2}$.

\section{Decay and Inverse Decay Rate}

Since we are interested in regimes where both the Higgs boson and the lepton momentum can be soft, we resum both propagators. The HTL resummation technique has been considered in Ref. 8 for the case of a Dirac fermion with Yukawa coupling.

In order to calculate the interaction rate $\Gamma$ of $N \leftrightarrow \ell \phi$, we cut the $N$ self energy and use the HTL resummation for the lepton and Higgs boson propagators. Since $\lambda_{\nu, i \alpha} \ll 1$, it is justified to neglect the coupling of the neutrino to the thermal bath. According to finite-temperature cutting rules, ${ }^{9 \mid 10}$ the interaction rate reads

$$
\Gamma(P)=-\frac{1}{2 p_{0}} \operatorname{tr}[(\not P+M) \operatorname{Im} \Sigma(P)] .
$$

At finite temperature, the self-energy reads

$$
\Sigma(P)=-g^{2} T \sum_{k_{0}=i(2 n+1) \pi T} \int \frac{\mathrm{d}^{3} k}{(2 \pi)^{3}} P_{L} S^{*}(K) P_{R} D^{*}(Q),
$$

where $P_{L}$ and $P_{R}$ are the projection operators on left- and right-handed states, $Q=P-K$ and we have summed over neutrino and lepton spins. We also sum over the two components of the doublets, particles and antiparticles and the three lepton flavors, such that $g^{2}=4\left(\lambda_{\nu}^{\dagger} \lambda_{\nu}\right)_{11}$.

The HTL-resummed Higgs boson propagator is $D^{*}(Q)=1 /\left(Q^{2}-m_{\phi}^{2}\right)$, where $m_{\phi}^{2} / T^{2}=\left(3 / 16 g_{2}^{2}+1 / 16 g_{Y}^{2}+1 / 4 y_{t}^{2}+1 / 2 \lambda\right)$ is the thermal mass of the Higgs boson. The couplings denote the $\mathrm{SU}(2)$ coupling $g_{2}$, the $\mathrm{U}(1)$ coupling $g_{Y}$, the top Yukawa coupling $y_{t}$ and the Higgs boson self coupling $\lambda$, where we assume a Higgs boson mass of $115 \mathrm{GeV}$. The other Yukawa couplings can be neglected since they are much smaller than unity and the remaining couplings are renormalized at the first Matsubara mode $2 \pi T$ as explained in Ref. 4 . 
The effective lepton propagator in the helicity-eigenstate representation is given by 111

$$
S^{*}(K)=\frac{1}{2} \Delta_{+}(K)\left(\gamma_{0}-\hat{\mathbf{k}} \cdot \gamma\right)+\frac{1}{2} \Delta_{-}(K)\left(\gamma_{0}+\hat{\mathbf{k}} \cdot \gamma\right),
$$

where

$$
\Delta_{ \pm}(K)=\left[-k_{0} \pm k+\frac{m_{\ell}^{2}}{k}\left( \pm 1-\frac{ \pm k_{0}-k}{2 k} \ln \frac{k_{0}+k}{k_{0}-k}\right)\right]^{-1}
$$

and $m_{\ell}^{2} / T^{2}=\left(3 / 32 g_{2}^{2}+1 / 32 g_{Y}^{2}\right)$.

The trace can be evaluated as

$$
\operatorname{tr}\left[(\not P+M) P_{L} S^{*}(K) P_{R}\right]=\Delta_{+}\left(p_{0}-p \eta\right)+\Delta_{-}\left(p_{0}+p \eta\right),
$$

where $\eta=\mathbf{p} \cdot \mathbf{k} / p k$ is the angle between neutrino and lepton. We evaluate the sum over Matsubara frequencies by using the Saclay method ${ }^{12}$ For the Higgs boson propagator, the Saclay representation reads

$$
D^{*}(Q)=-\int_{0}^{\beta} \mathrm{d} \tau e^{q_{0} \tau} \frac{1}{2 \omega_{q}}\left\{\left[1+n_{B}\left(\omega_{q}\right)\right] e^{-\omega_{q} \tau}+n_{B}\left(\omega_{q}\right) e^{\omega_{q} \tau}\right\},
$$

where $\beta=1 / T, n_{B}\left(\omega_{q}\right)=1 /\left(e^{\omega_{q} \beta}-1\right)$ is the Bose-Einstein distribution and $\omega_{q}^{2}=q^{2}+m_{\phi}^{2}$. For the lepton propagator it is convenient to use the spectral representation 13

$$
\Delta_{ \pm}(K)=-\int_{0}^{\beta} \mathrm{d} \tau^{\prime} e^{k_{0} \tau^{\prime}} \int_{-\infty}^{\infty} \mathrm{d} \omega \rho_{ \pm}(\omega, k)\left[1-n_{F}(\omega)\right] e^{-\omega \tau^{\prime}}
$$

where $n_{F}(\omega)=1 /\left(e^{\omega \beta}+1\right)$ is the Fermi-Dirac distribution and $\rho_{ \pm}$the spectral density 11

The lepton propagator in Eq. (5) has two different poles for $1 / \Delta_{ \pm}=0$, which correspond to two leptonic quasiparticles with a positive $\left(\Delta_{+}\right)$or negative $\left(\Delta_{-}\right)$ ratio of helicity over chirality 14$] 16$ The spectral density $\rho_{ \pm}$has a contribution from the poles and a discontinuous part. We are interested in the pole contribution

$$
\rho_{ \pm}^{\text {pole }}(\omega, k)=\frac{\omega^{2}-k^{2}}{2 m_{\ell}^{2}}\left(\delta\left(\omega-\omega_{ \pm}\right)+\delta\left(\omega+\omega_{\mp}\right)\right),
$$

where $\omega_{ \pm}$are the dispersion relations for the two quasiparticles, i.e. the solutions for $1 / \Delta \pm\left(\omega_{ \pm}, \mathbf{k}\right)=0$, shown in Fig. 1 (a). An analytical solution for $\omega_{ \pm}$can be found in the appendix of Ref. 1. One can assign a momentum-dependent thermal mass $m_{ \pm}(k)^{2}=\omega_{ \pm}(k)^{2}-k^{2}$ to the two modes as shown in Fig. 1 (b) and for very large momenta the heavy mode $m_{+}$approaches $\sqrt{2} m_{\ell}$, while the light mode becomes massless.

After evaluating the sum over $k_{0}$, carrying out the integrations over $\tau$ and $\tau^{\prime}$ and integrating over the pole part of $\rho_{ \pm}$in Eq. (10), we get

$$
\begin{aligned}
T \sum_{k_{0}} D^{*} \Delta_{ \pm}=-\frac{1}{2 \omega_{q}} & \left\{\frac{\omega_{ \pm}^{2}-k^{2}}{2 m_{\ell}^{2}}\left[\frac{1+n_{B}-n_{F}}{p_{0}-\omega_{ \pm}-\omega_{q}}+\frac{n_{B}+n_{F}}{p_{0}-\omega_{ \pm}+\omega_{q}}\right]\right. \\
& \left.+\frac{\omega_{\mp}^{2}-k^{2}}{2 m_{\ell}^{2}}\left[\frac{n_{B}+n_{F}}{p_{0}+\omega_{\mp}-\omega_{q}}+\frac{1+n_{B}-n_{F}}{p_{0}+\omega_{\mp}+\omega_{q}}\right]\right\},
\end{aligned}
$$


where $n_{B}=n_{B}\left(\omega_{q}\right)$ and $n_{F}=n_{F}\left(\omega_{ \pm}\right)$or $n_{F}\left(\omega_{\mp}\right)$, respectively.

The four terms in Eq. (11) correspond to the processes with the energy relations indicated in the denominator, i.e. the decay $N \rightarrow \phi \ell$, the production $N \phi \rightarrow \ell$, the production $N \ell \rightarrow \phi$ and the production of $N \ell \phi$ from the vacuum, as well as the four inverse reactions $\frac{9}{9}$ We are only interested in the process $N \leftrightarrow \phi \ell$, where the decay and inverse decay are illustrated by the statistical factors $1+n_{B}-n_{F}=$ $\left(1+n_{B}\right)\left(1-n_{F}\right)+n_{B} n_{F}$, given by the first term of Eq. (11).

For carrying out the integration over the angle $\eta$, we use

$$
\operatorname{Im} \frac{1}{p_{0}-\omega_{ \pm}-\omega_{q}}=-\pi \delta\left(p_{0}-\omega_{ \pm}-\omega_{q}\right)=-\pi \frac{\omega_{q}}{k p} \delta\left(\eta-\eta_{ \pm}\right) .
$$

After integrating over $\eta$ we get

$$
\begin{aligned}
\Gamma(P) & =\frac{1}{16 \pi p_{0} p} \sum_{ \pm} \int_{-1 \leq \eta_{ \pm} \leq 1} \mathrm{~d} k \frac{k}{\omega_{ \pm}}\left|\mathcal{M}_{ \pm}(P, K)\right|^{2}\left[1+n_{B}\left(\omega_{q \pm}\right)-n_{F}\left(\omega_{ \pm}\right)\right] \\
& =\frac{1}{2 p_{0}} \int \mathrm{d} \tilde{k} \mathrm{~d} \tilde{q}(2 \pi)^{4} \delta^{4}(P-K-Q)\left|\mathcal{M}_{ \pm}(P, K)\right|^{2}\left[1+n_{B}-n_{F}\right]
\end{aligned}
$$

where $\omega_{q \pm}=p_{0}-\omega_{ \pm}$, we only integrate over regions with $-1 \leq \eta \leq 1, \mathrm{~d} \tilde{k}=$ $\mathrm{d}^{3} k /\left((2 \pi)^{3} 2 k_{0}\right)$ and $\mathrm{d} \tilde{q}$ analogously and the matrix elements are

$$
\left|\mathcal{M}_{ \pm}(P, K)\right|^{2}=g^{2} \frac{\omega_{ \pm}^{2}-k^{2}}{2 m_{\ell}^{2}} \omega_{ \pm}\left(p_{0} \mp p \eta_{ \pm}\right)
$$

In order to compare our result to the conventional approximation, $\frac{4}{4}$ we do the same calculation for an approximated lepton propagator $S_{\text {approx }}^{*}(K)=1 /\left(\not K-m_{\ell}\right)$. This amounts to setting $\omega^{2}=k^{2}+m_{\ell}^{2}, \omega_{q}=p_{0}-\omega$ and we get $|\mathcal{M}|^{2}=\frac{g^{2}}{2}\left(M^{2}+\right.$ $\left.m_{\ell}^{2}-m_{\phi}^{2}\right)$ as matrix element.

This result resembles the zero temperature result with zero temperature masses $m_{\ell}, m_{\phi}$. The missing factor $1+n_{B}-n_{F}=\left(1+n_{B}\right)\left(1-n_{F}\right)+n_{B} n_{F}$ accounts for

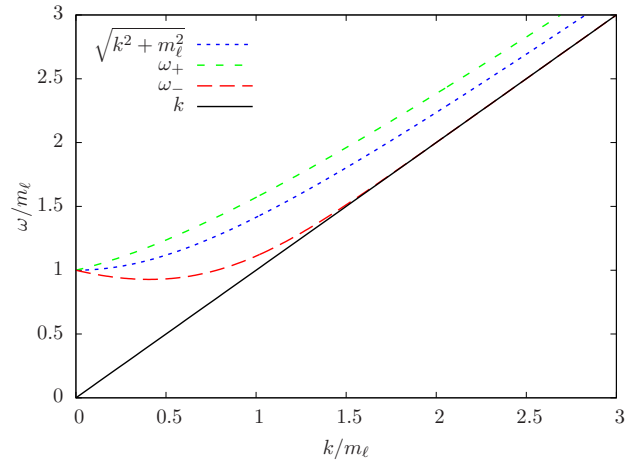

(a)

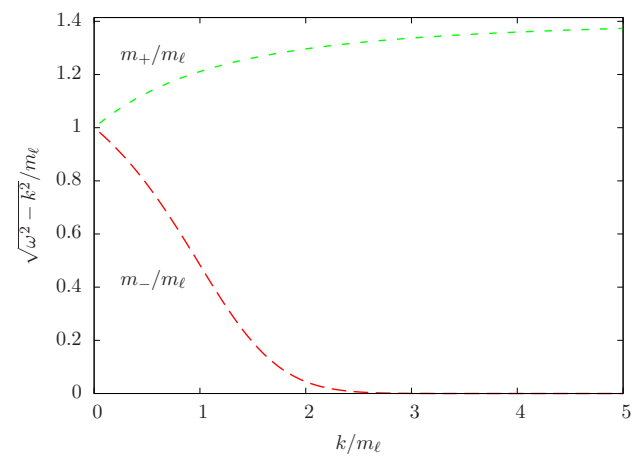

(b)

Fig. 1. (a) The two leptonic dispersion relations compared with the standard dispersion relation. (b) The momentum-dependent quasiparticle masses $m_{ \pm}^{2}=\omega_{ \pm}^{2}-k^{2}$. 
the statistical distribution of the initial or final particles. As pointed out in more detail in Ref. 17, we have shown that the approach to treat thermal masses like zero temperature masses in the final state ${ }^{4}$ is justified since it equals the HTL treatment with an approximate lepton propagator. However this approach does not equal the full HTL result.

\section{Decay Density}

The quantity which enters the Boltzmann equations is the decay density integrated over all neutrino momenta. In equilibrium it reads

$$
\gamma_{D}^{\mathrm{eq}}=\int \frac{\mathrm{d}^{3} p}{(2 \pi)^{3}} f_{N}^{\mathrm{eq}}(E) \Gamma_{D}=\frac{1}{2 \pi^{2}} \int_{M}^{\infty} \mathrm{d} E E p f_{N}^{\mathrm{eq}} \Gamma_{D},
$$

where $E=p_{0}, f_{N}^{\mathrm{eq}}(E)=[\exp (E \beta)-1]^{-1}$ is the equilibrium distribution of the neutrinos and $\Gamma_{D}=\left[1-f_{N}^{\mathrm{eq}}(E)\right] \Gamma$.

In Fig. 2, we compare our result to the conventional approximation. ${ }^{4} \mathrm{We}$ evaluate the decay rate for $M_{1}=10^{10} \mathrm{GeV}$ and $\tilde{m}_{1}=\left(\lambda_{\nu} \lambda_{\nu}^{\dagger}\right)_{11} v^{2} / M_{1}=0.06 \mathrm{eV}$, where $v=174 \mathrm{GeV}$ is the vacuum expectation value of the Higgs field.

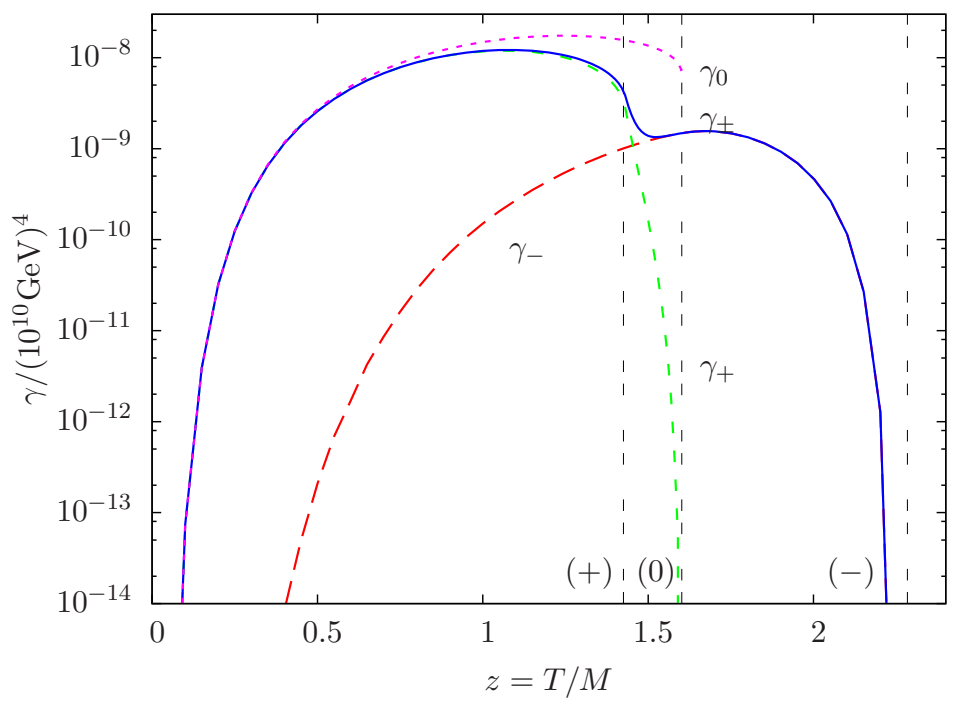

Fig. 2. The neutrino decay density with the one lepton mode approach $\gamma_{0}$ and the two-mode treatment $\gamma_{ \pm}$for $M_{1}=10^{10} \mathrm{GeV}$ and $\tilde{m}_{1}=0.06 \mathrm{eV}$. The thresholds for the two modes $(+),(-)$ and one mode (0) are indicated.

In the one-mode approach, the decay is forbidden when $M<m_{\ell}+m_{\phi}$. Considering two modes, the phase space is reduced for the positive mode due to the larger quasi-mass and at $M=m_{+}(\infty)+m_{\phi}$, the decay is only possible into leptons with small momenta, thus the rate drops dramatically. The decay into the negative, quasi-massless mode is suppressed due to its much smaller residue. However, 
the decay is possible up to $M=m_{\phi}$. These rates differ from the one mode approach by more than one order of magnitude in the interesting temperature regime of $z=T / M \gtrsim 1$.

\section{Conclusions}

As discussed in detail in Ref. 17, we have, by employing HTL resummation and finite temperature cutting rules, confirmed that treating thermal masses as kinematic masses as in Ref. 4 is a reasonable approximation. We have calculated the decay density of the lightest heavy Majorana neutrino and its behavior can be explained by considering the dispersion relations $\omega_{ \pm}$of the lepton modes and assigning momentum-dependent quasi-masses to them. The thresholds for neutrino decay reported in Ref. 4 are shifted and the decay density shows deviations of more than an order of magnitude in the interesting temperature regime $T / M \sim 1$. In order to arrive at a minimal consistent treatment, also the decay $\phi \rightarrow N \ell$ at high temperatures needs to be included as well as Higgs boson and neutrino CP asymmetries which are corrected for lepton modes.

This contribution summarizes the results of an earlier work ${ }^{1}$ and we refer the interested reader to the more elaborate treatment there.

Acknowledgements We thank Georg Raffelt, Florian Hahn-Wörnle, Steve Blanchet, Matthias Garny, Marco Drewes, Wilfried Buchmüller, Martin Spinrath and Philipp Kostka for fruitful and inspiring discussions.

\section{References}

1. C. P. Kießig, M. Plümacher and M. H. Thoma, arXiv:1003.3016 [hep-ph].

2. M. Fukugita and T. Yanagida, Phys. Lett. B 174, 45 (1986).

3. P. Minkowski, Phys. Lett. B 67, 421 (1977).

4. G. F. Giudice, A. Notari, M. Raidal, A. Riotto and A. Strumia, Nucl. Phys. B 685,89 (2004) arXiv:hep-ph/0310123.

5. M. Le Bellac, Thermal field theory (Cambridge University Press, Cambridge, UK, 1996).

6. E. Braaten and R. D. Pisarski, Nucl. Phys. B 337,569 (1990).

7. E. Braaten and R. D. Pisarski, Nucl. Phys. B 339,310 (1990).

8. M. H. Thoma, Z. Phys. C 66, 491 (1995). arXiv:hep-ph/9406242.

9. H. A. Weldon, Phys. Rev. D 28, 2007 (1983).

10. R. L. Kobes and G. W. Semenoff, Nucl. Phys. B 272, 329 (1986).

11. E. Braaten, R. D. Pisarski and T. C. Yuan, Phys. Rev. Lett. 64, 2242 (1990).

12. R. D. Pisarski, Nucl. Phys. B 309, 476 (1988).

13. R. D. Pisarski, Physica A 158, 146 (1989).

14. V. V. Klimov, Sov. J. Nucl. Phys. 33, 934 (1981) [Yad. Fiz. 33, 1734 (1981)].

15. H. A. Weldon, Phys. Rev. D 26, 2789 (1982).

16. H. A. Weldon, Phys. Rev. D 40, 2410 (1989).

17. C. P. Kießig and M. Plümacher, [arXiv:hep-ph/0910.4872]. 